\title{
Efficacy and Safety of Tiropramide in the Treatment of Patients With Irritable Bowel Syndrome: A Multicenter, Randomized, Double-blind, Non-inferiority Trial, Compared With Octylonium
}

\begin{abstract}
Kang Nyeong Lee, ${ }^{1}$ Oh Young Lee, ${ }^{1 *}$ Myung-Gyu Choi, ${ }^{2}$ Chong II Sohn, ${ }^{3}$ Kyu Chan Huh, ${ }^{4}$ Kyung Sik Park, ${ }_{1}^{5}$ Joong Goo Kwon, ${ }_{1}^{6}$ Nayoung Kim, ${ }^{7}$ Poong-Lyul Rhee, ${ }^{8}$ Seung-Jae Myung, ${ }^{9}$ Joon Seong Lee, ${ }^{10}$ Kwang Jae Lee, $^{11}$ Hyojin Park, ${ }^{12}$ Yong Chan Lee, ${ }^{12}$ Suck Chei Choi, ${ }^{13}$ Hye-Kyung Jung, ${ }^{14}$ Sam Ryong Jee, ${ }^{15}$ Chang Hwan Choi, ${ }^{16}$ Gwang Ha Kim, ${ }^{17}$ Moo In Park ${ }^{18}$ and In Kyung Sung ${ }^{19}$

${ }^{1}$ Department of Internal Medicine, Hanyang University College of Medicine, Seoul, Korea; ${ }^{2}$ Department of Internal Medicine, Catholic University College of Medicine, Seoul, Korea; ${ }^{3}$ Department of Internal Medicine, Kangbuk Samsung Medical Center, Sungkyunkwan University School of Medicine, Seoul, Korea; ${ }^{4}$ Department of Internal Medicine, Konyang University College of Medicine, Daejeon, Korea; ${ }^{5}$ Department of Internal Medicine, Keimyung University College of Medicine, Daegu, Korea; ${ }^{6}$ Department of Internal Medicine, Catholic University of Daegu, School of Medicine, Daegu, Korea; 'Department of Internal Medicine, Seoul National University Bundang Hospital, Seongnam, Korea; ${ }^{8}$ Department of Internal Medicine, Samsung Medical Center, Sungkyunkwan University School of Medicine, Seoul, Korea; ${ }^{9}$ Department of Internal Medicine, Asan Medical Center, University of Ulsan College of Medicine, Seoul, Korea; ${ }^{10}$ Department of Internal Medicine, Soonchunhyang University College, Seoul, Korea; ${ }^{11}$ Department of Internal Medicine, Ajou University College of Medicine, Suwon, Gyeonggi-do, Korea; ${ }^{12}$ Department of Internal Medicine, Yonsei University College of Medicine, Seoul, Korea; ${ }^{13}$ Department of Internal Medicine, Wonkwang University College of Medicine, Iksan, Korea; ${ }^{14}$ Department of Internal Medicine, Ewha Womans University College of Medicine, Seoul, Korea; ${ }^{15}$ Department of Internal Medicine, Inje University College of Medicine, Busan, Korea; ${ }^{16}$ Department of Internal Medicine, Chung-Ang University College of Medicine, Seoul, Korea; ${ }^{17}$ Department of Internal Medicine, Pusan University College of Medicine, Busan, Korea; ${ }^{18}$ Department of Internal Medicine, Kosin University College of Medicine, Busan, Korea; and ${ }^{19}$ Department of Internal Medicine, Konkuk University College of Medicine, Seoul, Korea
\end{abstract}

\section{Background/Aims}

Antispasmodics such as octylonium are widely used to manage irritable bowel syndrome (IBS) symptoms. However, the efficacy and safety of another antispasmodic, tiropramide, remain uncertain. We aimed to evaluate the efficacy and safety of tiropramide compared with octylonium in patients with IBS.

\section{Methods}

In this multicenter, randomized, non-inferiority trial, 287 patients with IBS (143 receiving tiropramide and 144 octylonium) were randomly allocated to either tiropramide $100 \mathrm{mg}$ or octylonium $20 \mathrm{mg}$ t.i.d (means 3 times a day) for 4 weeks. Primary

Received: October 7, 2013 Revised: November 5, 2013 Accepted: November 7, 2013

(c) This is an Open Access article distributed under the terms of the Creative Commons Attribution Non-Commercial License (http://creativecommons. org/licenses/by-nc/3.0) which permits unrestricted non-commercial use, distribution, and reproduction in any medium, provided the original work is properly cited.

*Correspondence: Oh Young Lee, MD, PhD

Department of Internal Medicine, Hanyang University Hospital, 222, Wangsimni-ro, Seongdong-gu, Seoul 133-791, Korea Tel: +82-2-2290-8343, Fax: +82-2-2298-8314, E-mail: leeoy@hanyang.ac.kr

Financial support: This work was supported by Dae Woong Pharma Korea Inc, Seoul, South Korea.

Conflicts of interest: None.

Author contributions: Kang Nyeong Lee analyzed data and wrote the manuscript. Oh Young Lee provided ideas, designed and supervised the manuscript. Myung-Gyu Choi, Chong II Sohn, Kyu Chan Huh, Kyung Sik Park, Joong Goo Kwon, Nayoung Kim, Poong-Lyul Rhee, Seung-Jae Myung, Joon Seong Lee, Kwang Jae Lee, Hyojin Park, Yong Chan Lee, Suck Chei Choi, Hye-Kyung Jung, Sam Ryong Jee, Chang Hwan Choi, Gwang Ha Kim, Moo In Park and In Kyung Sung designed and conducted the study. 
endpoint was the mean change of abdominal pain from baseline assessed by visual analogue scales (VAS) score after 4 weeks of treatment. Secondary endpoints were the changes in abdominal pain from baseline at week 2 and in abdominal discomfort at weeks 2 and 4, using VAS scores, patient-reported symptom improvement including stool frequency and consistency, using symptom diaries, IBS-quality of life (IBS-QoL), and depression and anxiety, at week 4.

\section{Results}

The VAS scores of abdominal pain at week 4, were significantly decreased in both tiropramide and octylonium groups, but the change from baseline did not differ between the 2 groups (difference, $-0.26 \mathrm{~mm} ; 95 \% \mathrm{Cl},-4.33-3.82 ; P=0.901$ ). Abdominal pain and discomfort assessed using VAS scores, diaries, and IBS-QoL were also improved by both treatments, and the changes from baseline did not differ. The incidence of adverse events was similar in the 2 groups, and no severe adverse events involving either drug were observed.

\section{Conclusions}

Tiropramide is as effective as octylonium in managing abdominal pain in IBS, with a similar safety profile.

(J Neurogastroenterol Motil 2014;20:113-121)

\section{Key Words}

Irritable bowel syndrome; Antispasmodic; Octylonium; Rome III criteria; Tiropramide

\section{Introduction}

Irritable bowel syndrome (IBS) is a functional bowel disorder characterized by abdominal pain or discomfort relieved by defecation and accompanied by a change in bowel habit. ${ }^{1}$ According to epidemiologic studies in Korea, the prevalence of IBS was reported to be $6.6 \%$ overall, $7.1 \%$ in men and $6.0 \%$ in women. ${ }^{2}$ Patients with IBS are reported to seek healthcare frequently, have poor health-related quality of life (QoL) and be responsible for high societal costs. ${ }^{3-5}$ Like other functional gastrointestinal (GI) disorders, the development of IBS and its symptoms are associated with multifactorial pathophysiology. ${ }^{6}$ Moreover, symptoms even in an individual patient with IBS may have a different pathophysiology depending on various factors such as diet or emotional status. Therefore, therapy targeting a specific pathophysiology in IBS has been difficult, and symptom treatment focusing on major complaints of IBS or drug combination covering multiple pathophysiology has usually been applied to the management of IBS.

Of multifactorial pathophysiology in IBS, colonic smooth muscle spasm has long been postulated to be responsible for abdominal pain in IBS from clinical observations as well as some experimental evidences. ${ }^{7}$ Therefore, pharmacologic agents directly acting on smooth muscle or on cholinergic receptors, called as an- tispasmodics, have been developed and used to control abdominal pain and discomfort in IBS. They may be classified as smooth muscle relaxants or agents with anti-cholinergic or anti-muscarinic property.

One of the antispasmodics, tiropramide, is a derivative of tyrosine, and exerts a spasmolytic effect on the intestine through reducing $\mathrm{Ca}^{2+}$ release into intestinal smooth muscle, rather than acting on the enteric nervous system. ${ }^{8,9}$ In a double-blind, placebo-controlled, randomized trial, tiropamide led to symptom improvement in terms of total symptom scores for 4 weeks, compared with 3 weeks in the placebo group; in addition, at week 4 abdominal pain was only improved in the tiropramide group. ${ }^{10}$ Also in a Korean study of similar design involving 63 patients with IBS, tiropramide improved overall symptom scores and decreased abdominal pain at weeks 2 and 4 (78.2\% of the treatment group with tiropramide and $40.5 \%$ of placebo group). ${ }^{10}$

Another antispasmodic, octylonium, also modifies $\mathrm{Ca}^{2+}$ fluxes in the intestinal smooth muscle, with direct myolytic properties similar to tiropramide. ${ }^{11,12}$ Previously, octylonium has already been proven to be more effective in reducing IBS symptoms than placebo. ${ }^{13-15}$ However, there have been few large-scale clinical trials comparing tiropamide with antispasmodics of proven efficacy in IBS patients. Therefore, this study aimed at evaluating the efficacy and safety profile of tiropamide in IBS patients compared with octylonium. 


\section{Materials and Methods}

\section{Study Patients}

Eligible patients were 20 to 75 years of age; had been diagnosed with IBS (as assessed according to the Rome III diagnostic criteria for IBS); complained of abdominal pain or discomfort at least 2 times per week for the 2 -week run-in period; had visual analogue scale (VAS) scores over $30 \mathrm{~mm}$ for abdominal pain during the randomization period. Patients who fit the study criteria had a run-in period of at least 2 weeks during which all IBS-related medications, and concomitant medications were stopped.

Exclusion criteria included a history of inflammatory bowel disease, lactose intolerance, uncontrolled diabetes (haemoglobin A1C $>8 \%$ ) or hypertension (systolic or diastolic blood pressure of $\geq 140 / 90 \mathrm{mmHg}$ ), psychiatric disorder requiring medication, malignancy of organs other than digestive (except for cases with no recurrence for over 5 years since treatment), laparoscopic or open abdominal surgery (except for appendectomy), renal or hepatic disease, glaucoma, and prostate hyperplasia. Those with a history of the following diseases within the previous 6 months were also excluded; peptic ulcer disease, GI bleeding, gastroesophageal reflux disease, intestinal stenosis or obstruction, infectious diarrhea, and pancreatic insufficiency. Patients were excluded if they were intolerable of or sensitive to, study drug, had a history of alcohol or drug addiction, or were expected to take any contraindicated medication during the study period. Pregnant or lactating women and women of childbearing age not using contraception, were also excluded.

\section{Study Design}

This was a multicenter (20 centers), randomized, double-blind, parallel-group, non-inferiority comparative study conducted in South Korea from May 2012 to April 2013. The study protocol was approved by the Institutional Review Board of each study center and conducted in accordance with the ethical principles based on the Declaration of Helsinki and Good Clinical Practice guidelines.

For a run-in period of about 2 weeks, eligible subjects for screening were instructed to stop IBS-related medications and prohibited from other drugs. Following the screening phase of 2 to 4 weeks, eligible patients were randomly assigned to either tiropramide (Tiropa; Dae Woong Pharma Korea Inc., Seoul, South Korea) $100 \mathrm{mg}$ or octylonium (Menoctyl; DONG HWA PHARM, Seoul, South Korea) $20 \mathrm{mg}$, taken 3 times daily before meals for 4 weeks. Treatment assignments were carried out by a computer-generated randomization schedule that was designed to allocate patients to the 2 treatment arms in a 1:1 ratio. The subjects were assigned to sequential allocation numbers at each site, and the medications were presented as 2 identically-appearing tablets containing 1 active drug and 1 placebo of each comparative drug to maintain the double blind condition. Follow-up visits during the study period were scheduled at weeks 2 and 4 to

\begin{tabular}{|c|c|c|}
\hline $\begin{array}{l}\text { Run-in } \\
\text { and } \\
\text { wash-out }\end{array}$ & $\begin{array}{l}\text { 4-week, } \\
\text { double-blind } \\
\text { treatment }\end{array}$ & $\begin{array}{c}\text { Tiropramide } \\
\text { or } \\
\text { octylonium }\end{array}$ \\
\hline
\end{tabular}

\begin{tabular}{|c|c|c|c|c|c|}
\hline $\begin{array}{c}\text { Visit } 1 \\
\text { screening }\end{array}$ & $\begin{array}{c}\text { Visit } 2 \\
\text { baseline }\end{array}$ & & Visit 3 & & Visit 4 \\
\hline 1 & $T$ & $T$ & T & $T$ & 7 \\
\hline$-4 \sim-2$ & 0 & 1 & 2 & 3 & 4 \\
\hline & & (wk) & & & \\
\hline
\end{tabular}

\begin{tabular}{|c|c|c|c|c|}
\hline \multicolumn{5}{|l|}{ Efficacy } \\
\hline VAS abdominal pain & & $\Delta$ & $\Delta$ & $\Delta$ \\
\hline VAS abdominal discomfort & & $\Delta$ & $\Delta$ & $\boldsymbol{\Delta}$ \\
\hline IBS-QoL & & $\Delta$ & & $\Delta$ \\
\hline $\mathrm{BDI}$ and $\mathrm{BAI}$ & & $\bar{\Delta}$ & & $\vec{\Delta}$ \\
\hline Symptom diary & $\longmapsto$ & & & -1 \\
\hline \multicolumn{5}{|l|}{ Safety } \\
\hline Adverse events & & $\Delta$ & $\Delta$ & $\Delta$ \\
\hline Physical examinations & $\Delta$ & $\Delta$ & $\Delta$ & $\Delta$ \\
\hline Vital signs & $\Delta$ & $\Delta$ & $\Delta$ & $\Delta$ \\
\hline Laboratory tests & $\Delta$ & & & $\vec{\Delta}$ \\
\hline
\end{tabular}

Figure 1. The study design. VAS, visual analogue scale; IBS-QoL, irritable bowel syndrome-quality of life; BDI, Beck depression inventory; BAI, Beck anxiety inventory. 
assess abdominal pain/discomfort using VAS, IBS quality of life, IBS symptoms including bowel habits using diaries, the Beck depression inventory (BDI) and the Beck anxiety inventory (BAI), drug compliance and adverse events (Fig. 1). Written, informed consent was obtained from all subjects before enrollment.

\section{Study Assessments}

\section{Efficacy}

The primary endpoint was the change in abdominal pain from baseline assessed by VAS scores after 4 weeks of treatment. The secondary endpoints included the change in abdominal pain from baseline by VAS scores at week 2, the change in abdominal discomfort from baseline by VAS scores at weeks 2 and 4, improvement of patients' overall assessment at weeks 2 and 4, the change in IBS QoL from baseline at 4 weeks, the change in symptom improvement in abdominal pain and discomfort from baseline using symptom diaries (severity of symptoms and days of no symptoms), and stool frequency and form assessed by Bristol stool form scale and according to IBS subtypes. Symptom improvement was defined as "completely improved," "considerably improved" or "slightly improved." Percentages of symptom im- provement include "completely, considerably and slightly improved." Symptom diaries assessed daily symptoms of abdominal pain and discomfort using VAS of 0 to 100 with 100 representing extreme symptom. The number of defecation per day and the most common stool consistency during the day were recorded using the Bristol stool form scale. IBS-QoL was assessed by asking patients to answer each question using a 5 -point Likert scale $(0=$ none, $1=$ mild, $2=$ moderate, $3=$ severe, $4=$ extremely severe). Exploratory end points were the changes in BDI and BAI from baseline. Each BDI or BAI was composed of 21 questions and assessed as " $0=$ do not feel at all," " $1=$ feel a bit," " $2=$ feel significantly," and " 3 = feel badly." Confounding factors were baseline factors of efficacy including BDI and BAI.

\section{Safety}

Safety assessments included adverse events, and abnormalities in laboratory findings and vital signs.

\section{Statistical Methods}

We estimated the required sample size for this study from the data of a previous study which found a change in VAS for abdominal pain from baseline as $23.6 \mathrm{~mm}$ with standard deviation

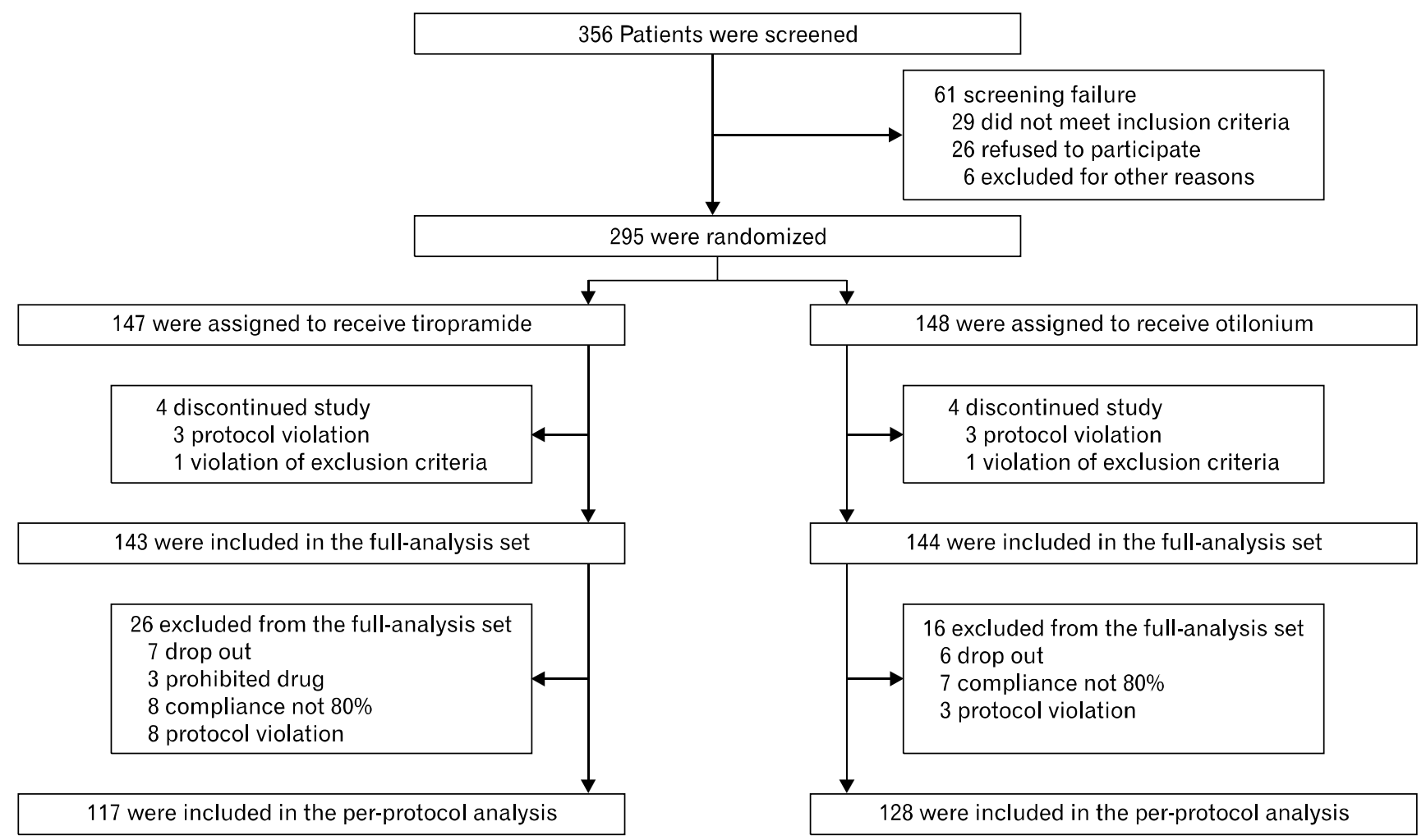

Figure 2. Enrollment, randomization and follow-up in the study. 
(SD) of $25.0 \mathrm{~mm} .{ }^{14}$ The margin of non-inferiority was assumed to be $11 \mathrm{~mm}$, approximately half of $22.7 \mathrm{~mm}$, which was the difference of the changes observed between the octylonium and the placebo groups. Based on these assumptions, a sample of 129 patients was needed for each study arm in order to have a power of $90 \%$, and a of 0.025 (one-sided), while allowing for a $15 \%$ dropout rate. The analyses were performed using full analysis set (FAS) and per protocol set (PPS).

All efficacy analyses were conducted in the FAS population, which consisted of all randomized patients satisfying the inclusion and exclusion criteria who received at least one dose of study medication and had at least one post-baseline efficacy measurement. The primary efficacy analysis was based on the PPS, which included all patients within the FAS population who took more than $80 \%$ of their assigned drugs and had no major protocol violations such as violations of scheduled visits or administration of contraindicated medication.

The change in VAS for abdominal pain from baseline was evaluated by 2 sample $t$ test. Seconday outcomes were compared by 2 sample $t$ test or Wilcoxon's rank sum test. Rates of symptom improvement were described as frequencies and percentages, and compared between groups by Pearson's chi-square test or Fisher's exact test. For safety variables including treatment emrgent adverse event (TEAE), adverse drug reaction, and serious adverse event, Pearson's chi-square test or Fisher's exact test was used to evaluate differences in incidence between groups. Changes in continuous variables from baseline after treatment were analyzed by 2 sample $t$ test or Wilcoxon's rank sum test,
Table 1. Demographic and Baseline Characteristics of the Full Analysis Set

\begin{tabular}{|c|c|c|c|}
\hline Characteristics & $\begin{array}{l}\text { Tiropramide } \\
(\mathrm{n}=143)\end{array}$ & $\begin{array}{l}\text { Octylonium } \\
(\mathrm{n}=144)\end{array}$ & $P$-value \\
\hline Age (yr) & & & $0.908^{\mathrm{a}}$ \\
\hline Mean \pm SD & $44.48 \pm 14.34$ & $44.76 \pm 14.90$ & \\
\hline $\begin{array}{l}\text { Median } \\
\text { (Min, Max) }\end{array}$ & $\begin{array}{c}43.00 \\
(20.00,72.00)\end{array}$ & $\begin{array}{c}44.00 \\
(20.00,73.00)\end{array}$ & \\
\hline $\operatorname{Sex}(\mathrm{n}[\%])$ & & & $0.595^{\mathrm{b}}$ \\
\hline Male & $68(47.55)$ & $73(50.69)$ & \\
\hline Female & $75(52.45)$ & $71(49.31)$ & \\
\hline $\mathrm{BMI}\left(\mathrm{kg} / \mathrm{m}^{2}\right)$ & & & $0.669^{\mathrm{a}}$ \\
\hline Mean $\pm \mathrm{SD}$ & $22.89 \pm 3.14$ & $23.00 \pm 3.01$ & \\
\hline $\begin{array}{l}\text { Median } \\
\text { (Min, Max) }\end{array}$ & $\begin{array}{c}22.60 \\
(16.77,33.50)\end{array}$ & $\begin{array}{c}22.70 \\
(16.91,30.86)\end{array}$ & \\
\hline $\begin{array}{l}\text { Examination } \\
\text { methods (n }[\%])\end{array}$ & & & $0.499^{\mathrm{c}}$ \\
\hline Colonoscopy & $143(100.00)$ & $142(98.61)$ & \\
\hline Colon study & $0(0.00)$ & $2(1.39)$ & \\
\hline $\begin{array}{c}\text { Duration of IBS } \\
\text { symptoms (yr) }\end{array}$ & & & $0.519^{\mathrm{a}}$ \\
\hline Mean \pm SD & $7.75 \pm 8.44$ & $8.11 \pm 7.97$ & \\
\hline $\begin{array}{l}\text { Median } \\
\text { (Min, Max) }\end{array}$ & $\begin{array}{c}5.04 \\
(0.56,49.62)\end{array}$ & $\begin{array}{c}5.70 \\
(0.53,48.30)\end{array}$ & \\
\hline $\begin{array}{l}\text { IBS subtypes } \\
(\mathrm{n}[\%])\end{array}$ & & & $0.913^{\mathrm{c}}$ \\
\hline IBS-C & $3(2.10)$ & $4(2.78)$ & \\
\hline IBS-D & $38(26.57)$ & $40(27.78)$ & \\
\hline IBS-M & $102(71.33)$ & $100(69.44)$ & \\
\hline
\end{tabular}

${ }^{a}$ Wilcoxon's rank sum test, ${ }^{b}$ Pearson's chi-square test, ${ }^{c}$ Fisher's exact test. BMI, body mass index; IBS, irritable bowel syndrome; IBS-C, constipation-predominant IBS; IBS-D, diarrhea-predominant IBS; IBS-M, mixed type IBS.

Table 2. Changes in Visual Analogue Scale Scores for Abdominal Pain From Baseline at Week 4

\begin{tabular}{|c|c|c|c|c|}
\hline VAS score (mm) & Tiropramide & Octylonium & Difference $(95 \% \mathrm{CI})$ & $P$-value \\
\hline PPS & $\mathrm{n}=117$ & $\mathrm{n}=128$ & & \\
\hline Baseline & $47.50 \pm 14.35$ & $48.24 \pm 12.82$ & & \\
\hline At week 4 & $24.02 \pm 14.99$ & $25.11 \pm 16.47$ & & \\
\hline Change & $-23.49 \pm 17.51$ & $-23.13 \pm 17.61$ & $-0.35(-4.78,4.07)$ & $0.875^{\mathrm{a}}$ \\
\hline In-group $P$-value & $<0.001^{\mathrm{b}}$ & $<0.001^{\mathrm{b}}$ & & \\
\hline FAS & $\mathrm{n}=143$ & $\mathrm{n}=144$ & & \\
\hline Baseline & $47.53 \pm 13.88$ & $48.00 \pm 12.78$ & & \\
\hline At week 4 & $24.49 \pm 15.20$ & $25.22 \pm 16.63$ & & \\
\hline Change & $-23.04 \pm 17.97$ & $-22.78 \pm 17.09$ & $-0.26(-4.33,3.82)$ & $0.901^{\mathrm{a}}$ \\
\hline In-group $P$-value & $<0.001^{\mathrm{b}}$ & $<0.001^{\mathrm{b}}$ & & \\
\hline
\end{tabular}

${ }^{\mathrm{a}}$ Two sample $t$ test, ${ }^{\mathrm{b}}$ Paired $t$ test.

VAS, visual analogue scale; PPS, per-protocol set; FAS, full-analysis set.

Change $=$ week $4-$ Baseline.

The upper limit of the $97.5 \% \mathrm{CI}$ at one-sided was $3.82 \mathrm{~mm}$, which is lower than the margin of non-inferiority of $11 \mathrm{~mm}$, indicating the non-inferiority of tiropramide to octylonium.

Values are means $\pm \mathrm{SD}$. 
while categorical variables, by Pearson's chi-square test or Fisher's exact test. Confounding factors such as BDI, BAI and baseline values of the efficacy variables were further analysed by ANCOVA or multiple logistic regression. A $P$-value of 0.05 was considered statistically significant. Statistical analyses were performed using SAS (Enterprise Guide 4.3).

\section{Results}

\section{Baseline Characteristics}

Total of 356 patients were evaluated for study inclusion. After exclusion of 61 patients during the screening period, 295 patients were randomized to either tiropramide or octylonium.

Table 3. The Change From Baseline in Secondary Endpoints

\begin{tabular}{|c|c|c|c|}
\hline & Tiropramide & Octylonium & $P$-value \\
\hline VAS score for abdominal pain & $\mathrm{n}=143$ & $\mathrm{n}=144$ & $0.752^{\mathrm{a}}$ \\
\hline Baseline & $47.53 \pm 13.88$ & $48.00 \pm 12.78$ & \\
\hline At week 2 & $33.03 \pm 17.06$ & $34.12 \pm 16.73$ & \\
\hline In-group $P$-value & $<0.001^{\mathrm{b}}$ & $<0.001^{\mathrm{b}}$ & \\
\hline VAS score for abdominal discomfort & $\mathrm{n}=143$ & $\mathrm{n}=144$ & \\
\hline Baseline & $52.57 \pm 15.61$ & $52.71 \pm 15.55$ & \\
\hline At week 2 & $38.68 \pm 19.65$ & $38.02 \pm 17.25$ & $0.729^{\mathrm{a}}$ \\
\hline At week 4 & $29.41 \pm 19.40$ & $29.30 \pm 17.42$ & $0.917^{\mathrm{a}}$ \\
\hline In-group $P$-value & $<0.001^{\mathrm{b}}$ & $<0.001^{\mathrm{b}}$ & \\
\hline Percentages of symptom improvement & $\mathrm{n}=143$ & $\mathrm{n}=144$ & \\
\hline At week 2 & $79.72 \%, 114 / 143$ & $74.31 \%, 107 / 144$ & $0.276^{\mathrm{c}}$ \\
\hline At week 4 & $86.33 \%, 120 / 139$ & $84.40 \%, 119 / 141$ & $0.648^{\mathrm{c}}$ \\
\hline In-group $P$-value & $<0.001^{\mathrm{b}}$ & $<0.001^{\mathrm{b}}$ & \\
\hline BDI & $\mathrm{n}=143$ & $\mathrm{n}=144$ & $0.080^{\mathrm{d}}$ \\
\hline Baseline & $14.83 \pm 9.27$ & $15.93 \pm 9.34$ & \\
\hline At week 4 & $11.97 \pm 9.12(4)$ & $11.47 \pm 8.57(3)$ & \\
\hline In-group $P$-value & $<0.001^{\mathrm{b}}$ & $<0.001^{\mathrm{b}}$ & \\
\hline BAI & $\mathrm{n}=143$ & $\mathrm{n}=144$ & $0.878^{\mathrm{d}}$ \\
\hline Baseline & $14.50 \pm 10.64$ & $13.37 \pm 8.76$ & \\
\hline At week 4 & $10.22 \pm 9.53(4)$ & $8.20 \pm 6.89$ & \\
\hline In-group $P$-value & $<0.001^{\mathrm{b}}$ & $<0.001^{\mathrm{b}}$ & \\
\hline IBS-QoL (total) & $\mathrm{n}=143$ & $\mathrm{n}=144$ & $0.070^{\mathrm{d}}$ \\
\hline Baseline & $77.66 \pm 27.57$ & $79.46 \pm 26.41$ & \\
\hline At week 4 & $61.96 \pm 24.07$ & $61.1 \pm 20.941(1)$ & \\
\hline In-group $P$-value & $<0.001^{\mathrm{b}}$ & $<0.001^{\mathrm{b}}$ & \\
\hline Stool frequency & $\mathrm{n}=38$ & $\mathrm{n}=40$ & $0.333^{\mathrm{a}}$ \\
\hline \multicolumn{4}{|l|}{ IBS-D } \\
\hline Baseline & $5.18 \pm 0.91$ & $5.14 \pm 0.85$ & \\
\hline At week 4 & $4.41 \pm 1.10(2)$ & $4.63 \pm 0.97(2)$ & \\
\hline In-group $P$-value & $<0.001^{\mathrm{b}}$ & $<0.001^{\mathrm{b}}$ & \\
\hline Stool consistency & $\mathrm{n}=38$ & $\mathrm{n}=40$ & $0.120^{\mathrm{d}}$ \\
\hline \multicolumn{4}{|l|}{ IBS-D } \\
\hline Baseline & $2.64 \pm 1.37$ & $2.27 \pm 1.36$ & \\
\hline At week 4 & $2.01 \pm 1.07(2)$ & $1.89 \pm 1.06(1)$ & \\
\hline In-group $P$-value & $<0.001^{\mathrm{b}}$ & $<0.032^{\mathrm{b}}$ & \\
\hline
\end{tabular}

${ }^{\mathrm{a}}$ Two sample $t$ test, ${ }^{\mathrm{b}}$ Paired $t$ test, ${ }^{\mathrm{c}}$ Pearson's chi-square test, ${ }^{\mathrm{d}}$ Wilcoxon rank sum test.

VAS, visual analogue scale; BDI, Beck depression inventory BAI, Beck anxiety inventory IBS-QoL, irritable bowel syndrome-quality of life IBS-D, diarrhea predominant IBS.

Values are means $\pm \mathrm{SD},($ ): number of dropout. Percentages of symptom improvement include "completely, considerably and slightly improved.” 
Table 4. Adverse Events During the Study

\begin{tabular}{lcc}
\hline \multicolumn{1}{c}{ Event } & $\begin{array}{c}\text { Tiropramide } \\
(\mathrm{n}=147)\end{array}$ & $\begin{array}{c}\text { Octylonium } \\
(\mathrm{n}=147)\end{array}$ \\
\hline Constipation & $3(2.04)$ & $2(1.36)$ \\
Dry mouth & $2(1.36)$ & $1(0.68)$ \\
Dyspepsia & $2(1.36)$ & $2(1.36)$ \\
Nausea & $1(0.68)$ & $2(1.36)$ \\
Headache & $2(1.36)$ & $1(0.68)$ \\
Chest pain & $0(0.00)$ & $2(1.36)$ \\
Nasopharyngitis & $2(1.36)$ & $0(0.00)$ \\
Myalgia & $1(0.68)$ & $2(1.36)$ \\
Serious adverse events & & \\
$\quad$ Idiopathic thrombocytopenic & $1(0.68)$ & $0(0.00)$ \\
$\quad$ purpura & & $1(0.68)$ \\
Intervertebral disc protrusion & $0(0.00)$ & \\
\hline
\end{tabular}

The adverse events listed were reported by 2 or more patients in either treatment group and the serious adverse events listed were reported in 1 patient from each treatment group.

Values are presented as $\mathrm{n}(\%)$

Three percent of patients in the tiropramide group (4/143) and octylonium group (4/144) did not complete the study. Data on the remaining 287 patients were available for the FAS analysis: 143 for tiropramide vs. 144 for octylonium. Data on 245 patients were available for the PPS analysis: 117 for tiropramide vs. 128 for octylonium. A flowchart of patient progression through the study with reasons for premature discontinuation is presented in Fig. 2. Demographic and baseline characteristics of patients are presented in Table 1. Both treatment arms were well balanced for gender, age and severity of symptoms at baseline.

\section{Efficacy}

The PPS population comprised of 245 patients (117 in the test group and 128 in the control group). The mean changes of VAS scores for abdominal pain from baseline were $-23.49 \pm$ $17.51 \mathrm{~mm}$ in the tiropramide group and $-23.13 \pm 17.61$ in the octylonium group. The mean difference between the 2 groups was $-0.26 \mathrm{~mm}$ in the FAS and $-0.35 \mathrm{~mm}$ in the PPS, and the upper limit of the $97.5 \% \mathrm{CI}$ at one-sided was $3.82 \mathrm{~mm}$ in the FAS and $4.07 \mathrm{~mm}$ in the PPS; these are below the margin of inferiority of $11 \mathrm{~mm}$, indicating that tiropramide is not inferior to octylonium (Table 2).

In secondary outcome analyses, VAS scores for abdominal pain at week 2 were significantly decreased in both groups (Table 3). Significant improvement in abdominal pain and discomfort at weeks 2 and 4 using symptom diaries, and in IBS-QoL, BDI and BAI at week 4 were observed in both groups, however the differ- ences between the groups were not significant. In the subgroup of IBS-D, stool frequency was decreased and stool consistency was improved by treatment. Furthermore, the degree of symptom reduction in abdominal pain using symptom diaries was higher in the tiropramide group than in the octylonium group from week 1 $(P=0.033)$.

After adjusting the baseline values of related factors, our analysis showed that all the factors based on symptom diaries, except the change of IBS symptoms, did not differ in the 2 groups. IBS symptoms at week 4 using symptom diaries were reduced further compared to baseline in the tiropramide group than the octylonium group.

\section{Safety}

Adverse events reported during the study are shown in Table 4. TEAE was $10.88 \%(16 / 147)$ in the tiropramide group and $12.24 \%(18 / 147)$ in the octylonium group, which included 28 each. The safety profile of tiropramide was similar to that of octylonium. Adverse drug reactions were reported in 7 patients in the tiropramide group and 6 patients in the octylonium group, which included 17 and 9, respectively. Serious adverse events developed in one patient in each group, idiopathic thrombocytopenic purpura in the tiropramide group and intervertebral disc protrusion in the octilonium group, but were not related to the study drugs, and were improved without any sequelae. Laboratory test results, when comparing those at screening period with those at last visit, showed no violation of safety regarding normal values, or the changes of direction and quantity. Vital signs did not change significantly from baseline in either group at weeks 2 and 4 .

\section{Discussion}

The present study was designed to assess the non-inferiority of tiropramide compared to the proven antispasmodic, octylonium, for managing IBS symptoms. This multicenter, randomized, double-blind, non-inferiority trial showed that 4-week treatment with tiropramide improved abdominal pain and discomfort, patient-reported overall assessments of IBS symptoms, IBS-QoL, depression and anxiety, with no significant differences from octylonium. These results demonstrate non-inferiority of tiropramide in efficacy and safety, compared with otiolonium.

Tiropramide effectively and safely managed abdominal pain in the IBS patients. Our results showed that the change in VAS scores for abdominal pain from baseline was about $23.5 \mathrm{~mm}$ after 
4-week treatment with tiropramide, similar to $23.6 \mathrm{~mm}$ observed after 4-week treatment with octylonium. The changes from baseline between the 2 groups were not significantly different, and the upper limit of $95 \% \mathrm{CI}$ was lower than the margin of inferiority in both the FAS and PPS populations, indicating non-inferiority of tiropramide compared to octylonium. In a previous study comparing tiroparmide with octylonium in IBS patients, the proportion of patients whose abdominal pain was relieved by treatment was higher in tiropramide group than in octylonium group at days 3 and 5 of medication, suggesting rapid drug action of tiropramide. ${ }^{16}$ Also, at the end of 30-day treatment, normal gut motility was restored in $88 \%$ of the tiropramide group compared with $47 \%$ of octylonium group. ${ }^{16}$

The presumed mechanism of tiropramide in improving abdominal pain is its antispasmodic effect on the intestine. In an animal study using the isolated smooth muscle, tiropramide inhibited $\mathrm{Ca}^{2+}$ influx for the most part and, to a lesser extent, increased the intracellular cyclic adenosine monophosphate (cAMP) levels. ${ }^{9}$ This study using rabbit also found that tiropamide increased $\mathrm{Ca}^{2+}$ absorption into microvesicles and decreased the amount of $\mathrm{Ca}^{2+}$ released to stimulate the contraction of the smooth muscle by increasing cAMP; as a consequence the smooth muscle relaxed. ${ }^{9}$ In terms of the distribution of tiropramide, a study showed that the radioactivity of tiropramide lasted longer in the colon than in other organs after intravenous or oral administration, ${ }^{17}$ indicating relative colonic selectivity. Furthermore, the effect of tiropramide were more evident in the smooth muscle of the digestive, urinary and female genital tract than in those of the vessel, and in stimulated contractions rather than in spontaneous ones. ${ }^{17}$

In the present study, other advantages of tiropramide were observed compared with octylonium: tiropramide was effective earlier than octylonium; in the assessment using symptom diaries, the severity and degree of abdominal discomfort tended to be reduced further in the tiropramide group than in the octylonium group. Since this study was designed to evaluate the non-inferiority of tiropramide over octylonium, we could not conclude that tiropramide is more effective than octylonium, but we may at least speculate about this possibility, considering that assessment using symptom diaries is less biased than that using VAS, which is based on patients' rememberance of symptoms from the previous week. Taken together, there may be some advantage of tiroparmide over octylonium for managing IBS symptoms; this could be due to its colonic selectivity.

Regarding the safety of tiropramide, percentages of patients reporting adverse events were similar in both groups. Vital signs and labortory test results did not significantly change after a 4-week treatment with either drug. It has been known that it does not affect neurotransmitters such as histamine, acetylcholine, and adrenaline, thus leading to a lower incidence of side effects than other antispasmodic agents. It was also found that tiropramide did not affect esophageal motility, including the pressure of the low esophageal sphincter, and the coordinated movements of the esophagus. ${ }^{18}$ Actually, both tiropramide and octylonium have been safely prescribed over the past ten years. Similarly, the present study raised no safety concern.

In the subgroup of patients with diarrhea predominant IBS (IBS-D), tiropramide improved bowel habits. Its spasmolytic effect of the drug may be related to the improvement of bowel habits in terms of stool frequency and consistency. These improvements may also be explained by the fact that tiropramide can restore normal intestinal transit in patients with IBS-D having rapid intestinal transit, and are consistent with observations in a study on octylonium demonstrating improvements of bowel habit and abdominal pain and discomfort. ${ }^{14}$ Moreover, as previously mentioned, gut motility was normalized in $88 \%$ of the tiropramide group after 30-day treatment with tiropramide, against $47 \%$ of the octylonium group. ${ }^{16}$ Considering the effect on bowel habit improvement in the subgroup of patients with IBS-D, there may be an additional benefit of tiropramide in patients with IBS-D, although the small number of patients with constipation predominant IBS (IBS-C) in our study might preclude us from concluding that the study drug is effective in patients with IBS-C.

In this study, levels of depression and anxiety in the IBS patients were also improved by tiropramide. This may be interpreted in terms of the brain-gut axis. ${ }^{19}$ Relief of abdominal pain may affect positively the emotional status of study patients. IBS-QoL was also improved. However, theses secondary outcomes should be cautiously interpreted because there may be a variety of confounding factors. Placebo effects are known to be relatively high in IBS, ranging from $20 \%$ to $70 \%$. This is believed to be partly due to a variety of physical and psychological factors affecting the brain-gut axis, which is thought to be involved in IBS pathophysiology. Therefore, studies of the treatment of IBS should be designed as randomized, double-blind, and placebo-controlled trials. ${ }^{20}$ The present study lacks a placebo control therefore could be limited in directly showing the superiority of tiropramide to placebo. However, this limitation in our study may be sufficiently compensated by previous evidence to demonstrate 
the superiority of octylonium to placebo. ${ }^{13,14,21}$ Other limitations of our study include the relatively short period of treatment (only 4 weeks) and the lower dose of octylonium than that used in the reference study (20 mg vs. $40 \mathrm{mg}$, t.i.d). However, the efficacy of both tiropramide and octylonium in improving symptoms of IBS was clearly demonstrated by our study results with statistical differences between pre- and post-treatments.

In conclusion, our study demonstrates that treatment with tiropramide is effective and safe for managing IBS symptoms such as abdominal pain and discomfort, stool frequency and consistency, IBS-QoL, depression and anxiety. Considering not enough data fully show the efficacy and safety of tiropramide in IBS patients, the present study should be a useful reference in IBS research and clinical practice for managing IBS patients. Further studies are required to elucidate the mechanism of action of tiropramide on intestine, particularly its pharmacodynamic and pharmacokinetic properties .

\section{References}

1. Longstreth GF, Thompson WG, Chey WD, Houghton LA, Mearin F, Spiller RC. Functional bowel disorders. Gastroenterology 2006;130:1480-1491.

2. Han SH, Lee OY, Bae SC, et al. Prevalence of irritable bowel syndrome in Korea: population-based survey using the Rome II criteria. J Gastroenterol Hepatol 2006;21:1687-1692.

3. Hillilä MT, Färkkilä NJ, Färkkilä MA. Societal costs for irritable bowel syndrome - a population based study. Scand J Gastroenterol 2010;45:582-591.

4. Gralnek IM, Hays RD, Kilbourne A, Naliboff B, Mayer EA. The impact of irritable bowel syndrome on health-related quality of life. Gastroenterology 2000;119:654-660.

5. Koloski NA, Talley NJ, Boyce PM. Epidemiology and health care seeking in the functional GI disorders: a population-based study. Am J Gastroenterol 2002;97:2290-2299.

6. Barbara G, De Giorgio R, Stanghellini V, Cremon C, Salvioli B, Corinaldesi R. New pathophysiological mechanisms in irritable bowel syndrome. Aliment Pharmacol Ther 2004;20(suppl 2):1-9.

7. American College of Gastroenterology Task Force on Irritable Bowel Syndrome, Brandt LJ, Chey WD, et al. An evidence-based position statement on the management of irritable bowel syndrome. Am J Gastroenterol 2009;104(suppl 1):S1-S35.
8. Takayanagi I, Hisayama T, Iwase M, Sakuma N, Nagai H. Pharmacological properties of tiropramide, an antispasmodic drug. Gen Pharmacol 1989;20:335-339.

9. Uruno T, Shirane M, Wada K, et al. Possible mechanisms of action of the antispasmodic agent tiropramide in the isolated detrusor from rats. Jpn J Pharmacol 1992;60:275-280.

10. Park SH, Jang CH, Han JY, et al. [Double blind clinical trial of tiropramide in irritable bowel syndrome.] Korean J Gastroenterol 1993; 25:877-883. [Korean]

11. Gallego D, Aulí M, Aleu J, et al. Effect of otilonium bromide on contractile patterns in the human sigmoid colon. Neurogastroenterol Motil 2010;22:e180-e191.

12. Strege PR, Evangelista S, Lyford GL, Sarr MG, Farrugia G. Otilonium bromide inhibits calcium entry through L-type calcium channels in human intestinal smooth muscle. Neurogastroenterol Motil 2004; 16:167-173.

13. Baldi F, Corinaldes R, Ferrarini F, et al. Clinical and functional evaluation of octilonium bromide in the treatment of irritable bowel syndrome. A double blind controlled trial. Clin Trials J 1983;20:77-88.

14. Glende M, Morselli-Labate AM, Battaglia G, Evangelista S. Extended analysis of a double-blind, placebo-controlled, 15-week study with otilonium bromide in irritable bowel syndrome. Eur J Gastroenterol Hepatol 2002;14:1331-1338.

15. Clave $\mathrm{P}$, Acalovschi M, Triantafillidis JK, et al. Randomised clinical trial: otilonium bromide improves frequency of abdominal pain, severity of distention and time to relapse in patients with irritable bowel syndrome. Aliment Pharmacol Ther 2011;34:432-442.

16. Tinozzi S, Valesi MG. [Controlled clinical study on the efficacy of tiropramide hydrochloride in the treatment of irritable colon: comparison with octylonium bromide.] Minerva Med 1984;75:23-31. [Italian]

17. Setnikar I, Cereda R, Pacini MA, Revel L, Makovec F. Pharmacological characterisation of the smooth muscle antispasmodic agent tiropramide. Arzneimittelforschung 1989;39:1114-1119.

18. Bolognese A, Lamazza A, Tocchi A, et al. [Tiropramide chlorhydrate in premedication for endoscopic retrograde cholangiopancreatography and its effects on the motor activity of Oddi's sphincter.] Minerva Med 1987;78:303-306. [Italian]

19. Tanaka Y, Kanazawa M, Fukudo S, Drossman DA. Biopsychosocial model of irritable bowel syndrome. J Neurogastroenterol Motil 2011; 17:131-139.

20. Klein KB. Controlled treatment trials in the irritable bowel syndrome: a critique. Gastroenterology 1988;95:232-241.

21. Poynard T, Regimbeau C, Benhamou Y. Meta-analysis of smooth muscle relaxants in the treatment of irritable bowel syndrome. Aliment Pharmacol Ther 2001;15:355-361. 